\title{
La Barranca del Rio Santiago como atractivo turístico y ecoturístico para la marca cuidad Guadalajara Guadalajara, enfocado a un turismo nacional
}

\section{The ravine of the Santiago River as tourist and ecotourism attraction for the Guadalajara, Guadalajara city brand, focused on national tourism}

José G. Vargas-Hernández¹, Jovanna Nathalie Cervantes-Guzmán², Elba Lizbeth García Guerra³

Resumen: El propósito principal de esta investigación es exponer si la Barranca del Rio Santiago es un atractivo turístico y ecoturismo para que se incluya en la marca Guadalajara, enfocado al turismo nacional. La investigación es de carácter exploratorio y descriptivo, pues se busca saber si la barranca del Rio de Santiago es un atractivo turístico y ecoturístico para que se pueda incluir en la marca Guadalajara. Se realizó el análisis bajo una base de datos emitida por el estado de Jalisco, el cual es el Anuario del 2016 del Estado de Jalisco. En la investigación solo se tomaron los indicadores nacionales de la Zona Metropolitana de Guadalajara en referencia a motivación, lo que más les gusta, medio por el que llegan al destino, etc. Se concluye que SECTUR establece en la última agenda de competitividad de los destinos turístico de México que Guadalajara cuenta con una gran cantidad de destinos naturales, entre ellos la Barranca del Río Santiago, este destino cuenta con un alto valor ecológico, recreativo y de paisajismo, el cual es muy poco conocido por la población local, por consecuente puede llegar a ser un nuevo producto para el sector ecoturístico.

Palabras claves: City marketing, Marca cuidad, Ecoturismo, Guadalajara, Guadalajara.
Abstract: The main purpose of this research is to show if the Barranca del Rio Santiago is a tourist attraction and ecotourism to be included in the Guadalajara brand, focused on national tourism. The research is exploratory and descriptive in nature, as it seeks to know if the Rio de Santiago canyon is an ecotourism and tourist attraction so that it can be included in the Guadalajara brand. The analysis was conducted under a database issued by the state of Jalisco, which is the 2016 Yearbook of the State of Jalisco. In the research, only the national indicators of the Metropolitan Area of Guadalajara were taken in reference to motivation, what they like most, means by which they arrive at the destination, etc. As conclusion, SECTUR establishes in the latest competitiveness agenda of tourist destinations in Mexico that Guadalajara has a large number of natural destinations, including the Barranca del Río Santiago, this destination has a high ecological, recreational and landscaping value, which is very little known by the local population, and consequently it can become a new product for the ecotourism sector.

Keywords: City marketing, City brand, Ecotourism, Guadalajara, Guadalajara.

(Presentado: 19 de septiembre de 2018. Aceptado: 10 de septiembre de 2019).

\footnotetext{
1 Profesor investigador, Centro Universitario de Ciencias Económico Administrativas, Universidad de Guadalajara, México. jvargas2006@ggmail.com

${ }^{2}$ Asistente de investigación, Centro Universitario de Ciencias Económico Administrativas, Universidad de Guadalajara, México. nathalie.cervantes34Qిoutlook.com

${ }^{3}$ Asistente de investigación, Centro Universitario de Ciencias Económico Administrativas, Universidad de Guadalajara, México. nathalie.cervantes34@ioutlook.com
} 


\section{INTRODUCCIÓN}

En la siguiente investigación se analiza si la Barranca del Río Santiago puede ser un atractivo turístico y ecoturístico para la marca cuidad de Guadalajara, enfocado en un turismo nacional. Por ello se habla de los términos turismo, turismo alternativo, turismo nacional, ecoturismo, que es un atractivo turístico, la marca cuidad o city marketing, la marca Guadalajara Guadalajara y la Barranca del Río Santiago. Todos estos términos son basados en varios autores expertos en la materia y documentos oficiales los cuales los proporciona la Secretaria de Turismo del Estado de Jalisco.

Sin duda alguna, las marcas ciudades son algo que se viene planteando desde hace mucho tiempo y que existen casos de éxito muy famosos, como lo es I Love NY o lo que es la marca CDMX (Aldaz, 2016). Otro aspecto importante de la investigación es que la marca Guadalajara Gudalajara, fue lanzada por el Gobierno de Guadalajara en el 2016 con la característica canción del compositor Pepe Guízar (Comunicación social, eventos, gobierno noticias 2016).

En cuanto a los temas turísticos se comenta que, "El turismo es una fuerza económica, que tiene el poder de implementar proyectos que mantengan el atractivo natural que los visitantes desean ver y experimentar. Los beneficios económicos del turismo dependen de la manera en que se mantengan los atractivos turísticos seguros, limpios y agradables a los sentidos" (Rebollo, 2012, p.). El turismo alternativo se refiere "a que existen otras formas de hacer las cosas. Por ejemplo, el viajar y conocer lugares, más naturales" (Rogel, Rojas \&Ortega, 2011, (p.).

Sin embargo, hay que definir perfectamente qué fracción de turistas se van a tomar del turismo nacional, que es el que practican los nacionales y extranjeros residentes en el país dentro de las fronteras de éste, es decir, los turistas que se desplazan en el interior del país lo hacen por motivos vacacionales, recreación, congresos y convenciones, trabajo y negocios (Portugal, 2008). Para después poder segmentarlo más y poder llegar al sector ecoturístico el cual se tiene como "el resultado directo de la aceptación mundial a favor de prácticas productivas más sostenibles y con menor impacto al medio ambiente".

\section{PROBLEMA DE INVESTIGACIÓN}

\section{Planteamiento del problema}

El city marketing es un suceso que ha generado debates, pues por una parte se señala que la comercialización de territorios existe desde hace siglos, y por otra parte, se sostienen que es una tendencia que surge a partir de las décadas de los setenta y ochenta (Armas, 2007). Sin embargo, tenemos otros autores que comentan que "El marketing de ciudad (city marketing) se puede definir como política activa de actuaciones orientadas, por una parte, a identificar y determinar las necesidades de sus diferentes públicos, reales y potenciales; y otra parte a desarrollar una serie de productos y servicios en la ciudad para satisfacer dichas necesidades, creando y potenciado su demanda" (Rojano \& Castilla, 2007).

"Se conocen casos de éxito de las marcas ciudades, por ejemplo, I love NY" (Marín, 2010). En México actualmente existe la marca Vistit México (CPTM, 2017), sin embargo, Guadalajara quiso realizar su propia marca cuidad la cual lleva el slogan "Guadalajara, Guadalajara" creada en el 2016 como un elemento de identidad, herramienta de promoción y posicionamiento de la ciudad de Guadalajara" (Comunicación social, eventos, Gobierno, noticias, 2016). Los creadores de la marca "se basaron en la canción de Pepe Guízar, puesto que representa un símbolo natural de la cuidad y refuerza emociones e ideas ya posicionadas, con el característico coro Guadalajara, Guadalajara. De igual manera se realizó como publicidad la adaptación de la canción del compositor antes mencionado, en la cual se mezcló diferentes estilos de música como lo es el mariachi, rock, electrónica, jazz y música regional, también se mezcló una variedad de cantantes con la intención de integrar a todo México (Cardona, 2016).

Un factor importante es la intervención del sector turístico. Troisi define turismo como "El conjunto de traslados temporales de las personas originado por necesidades de reposo, cura, espirituales o intelectuales" (Betancourt, 2008). Un factor importante es tomar en cuenta el turismo nacional el cual se define como "los turistas que se desplazan en el interior del país lo hacen por motivos vacacionales, recreación, congresos y convenciones, trabajo y/o negocios, etc.” (Portugal, 2008). 
También es importante señalar que, según Ipsos el tracking publicitario del consejo de promoción turística preliminar, tomando en cuenta 3 indicadores los cuales nos mencionan que "en el 2017 el $46 \%$ de personas visitan un lugar son por atribución publicitarias; $90 \%$ son personas que intención de viaje y el tercer indicador por conocimiento de marca el cual de manera nacional no tiene ningún porcentaje. De igual manera "una encuesta nacional sobre Orgullos del Mexicano en 2016 realizada por la consultora Mitofsky menciona que el 9.9\% de los 1000 encuestados mayores de 18 años contestó que Guadalajara era la primera ciudad donde más orgullosos se sentían los mexicanos" (Consultora Mitofsky, 2016).

Según expertos que elaboraron la agenda competitiva de destinos turísticos de México señala que "México no ha sabido aprovechar el total de beneficios que brinda la actividad turística, un claro ejemplo de esto es la ciudad de Guadalajara la cual es uno de los estados emblemáticos de México, esta cuidad es destacada por su gran aportación cultural, tradición y edificaciones históricas, sin embargo, no ha sabido aprovechar, el patrimonio natural que tiene para darle un uso recreativo o turístico, puesto que cuenta con un gran potencial en este sector como lo es la Barranca del Río Santiago" (SECTUR, 2014).

Tras todos estos datos proporcionados se genera un vacío el cual se convierte en el propósito de la investigación que consiste en saber que tan atractivo puede ser el ecoturismo para que pueda ser agregado a la marca Guadalajara, Guadalajara y cuáles pueden ser las zonas potenciales. Con la intención de aportar este estudio a las autoridades correspondientes en el sector turístico, para la ampliación del mercado laboral del sector turístico de una manera que sean capacitados para poder realizar actividades ecoturísticas. Tomando en cuenta que la definición de atractivo turístico, el diccionario de terminología turística de la Dirección Nacional de Turismo Argentina menciona "que un atractivo turístico es el elemento natural, cultural, deportivo o de cualquier otro tipo que pueda generar suficiente interés para atraer turistas" (Navarro, 2015).

\section{Justificación}

En esta investigación se plantea saber si la Barranca del Río Santiago es un atractivo turístico y ecoturístico para la marca cuidad Guadalajara Guadalajara, con el fin de poder impulsar que se pueda tener un uso recreativo y turístico de las zonas de la ciudad de Guadalajara que no soy muy conocidas, considerando que puede tener un mayor atractivo para el turismo nacional, puesto que la proximidad de estas zonas puede ser de gran atractivo para personas que habitan en la ciudad de Guadalajara y tienen el gusto para disfrutar de ambientes naturales cerca de ellos. También se pretende que pueda servir como apoyo el desarrollo de nuevos programas ya sea para Secretaria de Turismo (SECTUR).

\section{OBJETIVOS}

\section{Objetivos generales}

Determinar la factibilidad de que la barranca del río Santiago sea un atractivo turístico y ecoturístico para la marca Guadalajara, Guadalajara, para el turismo nacional.

\section{Objetivos específicos}

- Exponer lugares de atractivo turístico de Guadalajara para usos recreativos.

- Exponer el perfil del ecoturista.

- Exponer la capacitación de personal calificado para atender a los ecoturistas nacionales.

\section{HIPÓTESIS}

\section{Hipótesis general}

¿Es factible que la barranca del río Santiago sea un atractivo turístico y ecoturismo para la marca Guadalajara, con un enfoque al turismo nacional?

\section{Hipótesis especifica}

- ¿Exponer que la Barranca del río Santiago es un atractivo turístico y ecoturísticas en Guadalajara?

- ¿El sector ecoturístico es rentable para el turismo nacional?

- ¿Existe suficiente personal capacitado para el sector ecoturístico? 


\section{Matriz de operacionalidad de las variables}

Tabla No 1: Matriz de operacionalidad de las variables.

\begin{tabular}{|c|c|c|c|}
\hline Tema & Objetivos & Hipótesis & Variables \\
\hline $\begin{array}{l}\text { La Barranca del Rio } \\
\text { Santiago como atractivo } \\
\text { turístico y ecoturístico } \\
\text { para la marca cuidad } \\
\text { Guadalajara Guadalajara, } \\
\text { enfocado a un turismo } \\
\text { nacional }\end{array}$ & $\begin{array}{l}\text { Exponer lugares de atractivo } \\
\text { turístico de Guadalajara, } \\
\text { para usos recreativos. } \\
\text { Exponer el perfil del } \\
\text { ecoturista. } \\
\text { Exponer la capacitación } \\
\text { de personal calificado } \\
\text { para atender a los } \\
\text { ecoturistas nacionales }\end{array}$ & $\begin{array}{l}\text { Exponer que la Barranca } \\
\text { del rio Santiago es un } \\
\text { atractivo turístico y } \\
\text { ecoturísticas en Guadalajara } \\
\text { El sector ecoturístico } \\
\text { es rentable para el } \\
\text { turismo nacional } \\
\text { Existe suficiente personal } \\
\text { capacitado para el } \\
\text { sector ecoturístico }\end{array}$ & $\begin{array}{l}\text { Ecoturismo } \\
\text { Turismo nacional }\end{array}$ \\
\hline
\end{tabular}

Fuente: Elaboración propia.

\section{REVSIÓN DE LITERATURA}

Tabla Nº 2: Revisión de la literatura.

\begin{tabular}{lllll}
\hline Autor & Año & Muestra & País & Factores empíricos \\
\hline Yesid Betancourt & 2008 & $\begin{array}{l}\text { La muestra fue de 700 } \\
\text { viajeros internacionales }\end{array}$ & Colombia & City Maketing. \\
Sectur & 2014 & - & México & Barranca del Rio Santiago. \\
Rafael Guerrero & 2010 & - & México & Ecoturismo. \\
Diego Navarro & 2015 & - & España & Atractivos turisticos. \\
\hline
\end{tabular}

Fuente: Elaboración propia.

\section{MARCO CONTEXTUAL}

\section{Turismo}

El turismo es "la suma de los fenómenos y relaciones que surgen del viaje y de la permanencia de los no residentes, mientras no establezcan una residencia permanente y no se relacionen con cualquier actividad remunerada", esta definición fue a cargo de los profesores Hunziker y Krapf en 1942. De igual manera Troisi lo define como "el conjunto de traslados temporales de las personas originado por necesidades de reposo, cura, espirituales o intelectuales" (Betancourt, 2008). Posteriormente, autores como Burkart y Medlik en el año 1981 definen que son "los desplazamientos cortos y temporales de la gente hacia destinos fuera del lugar de residencia y de trabajo, y las actividades emprendidas durante la estancia en esos destinos" (Sancho, 2008).
En 1982 por parte de Mathieson y Wall se menciona que "el turismo es el movimiento temporal de la gente, por períodos inferiores a un año, a destinos fuera del lugar de residencia y de trabajo, las actividades emprendidas durante la estancia y las facilidades creadas para satisfacer las necesidades de los turistas" (Sancho, 2008). El profesor Arriaga lo menciona como que "turismo es todo desplazamiento temporal determinado por causas ajenas al lucro: El conjunto de bienes, servicios y organización que en cada nación determinan y hacen posibles esos desplazamientos y las relaciones y hechos que entre éstos y los viajeros tienen lugar (Betancourt, 2008). Sin embargo, tras tantas definiciones la Organización Mundial del Turismo (OMT) en el año 1994 
lo define como: "el turismo comprende las actividades que realizan las personas durante sus viajes y estancias en lugares distintos al de su entorno habitual, por un período de tiempo consecutivo inferior a un año con fines de ocio, por negocios y otros” (Sancho, 2008).

En esta investigación se utilizará la definición de la OMT porque es la principal organización internacional en el ámbito turístico, contando con 158 países, 6 miembros asociados y más de 500 miembros privados (OMT, 2018). Autores como Óscar de la Torre Padilla en 1991, lo define como "el turismo es un fenómeno social que consiste en el desplazamiento voluntario y temporal de individuos o grupos de personas que, fundamentalmente con motivo de recreación, descanso cultura o salud, se trasladan de su lugar de residencia habitual a otro, en el que no ejercen ninguna actividad lucrativa ni remunerada, generando múltiples interrelaciones de importancia social, económica y cultural” (Betancourt, 2008).

"El turismo es una fuerza económica, que tiene el poder de implementar proyectos que mantengan el atractivo natural que los visitantes desean ver y experimentar. Los beneficios económicos del turismo dependen de la manera en que se mantengan los atractivos turísticos seguros, limpios y agradables a los sentidos" (Rebollo, 2012). Al igual que es importante el desarrollo turístico ha traído fuertes impactos en el medioambiente como la contaminación de ríos, acústica y estética. Aunque entre los impactos más importantes cabe citar la pérdida de la diversidad biológica y la limitación de recursos naturales sobre los cuales se asienta la actividad turística.

Esta sobreexplotación de recursos se ha incrementado en los últimos años a niveles no sostenibles. "El turismo no puede funcionar sin los recursos naturales básicos sobre los que se asienta ya que su propia degradación ambiental incide directamente sobre su oferta y puede poner en peligro la misma actividad turística en sí. Por todo ello diversos organismos y convenciones en los últimos años han tomado conciencia de la importancia de la conservación del entorno natural para aplicar no solo al sector turístico sino a todos los ámbitos sociales para alcanzar un desarrollo sostenible" (Betancourt, 2008). Conforme el turismo se va desarrollando, deberá hacer de la preservación del ambiente, una prioridad. La industria del turismo tendrá que proteger y preservar las atracciones turísticas naturales, el medio ambiente, los ecosistemas frágiles y las culturas vivas de regiones alejadas. De esta manera los turistas continuarán viajando” (Rebollo, 2012).

\section{Turismo alternativo}

El turismo alternativo es definido por la SEMARNAT en el 2004 como "los viajes que tienen como fin realizar actividades recreativas en contacto directo con la naturaleza y las expresiones culturales que le envuelven con una actitud y compromiso de conocer, respetar, disfrutar y participar en la conservación de los recursos naturales y culturales. El turismo alternativo es producto de las políticas internacionales para conservar el medio ambiente y es una respuesta en México a las políticas medioambientales que tienen como objetivo la conservación de los recursos naturales" (Rogel, Rojas \&Ortega, 2011). Al igual que Neyra en el 2004 menciona que el turismo alternativo se refiere "a que existen otras formas de hacer las cosas. Por ejemplo, el viajar y conocer lugares (turismo alternativo), no está exento de alternativas nuevas, en este concepto lo más importante es el contacto con la naturaleza y las culturas autóctonas, es decir empaparse de lo propio de la región a visitar" (Rogel, Rojas \&Ortega, 2011).

\section{Turismo nacional}

La Organización Mundial del turismo nos brinda la siguiente definición de turismo nacional "El turismo nacional abarca el turismo interno y el turismo emisor, a saber, las actividades realizadas por los visitantes residentes dentro y fuera del país de referencia, como parte de sus viajes turísticos internos o emisores" (OMT, 2008). También se puede tomar como definición que el turismo nacional "es el que practican los nacionales y extranjeros residentes en el país dentro de las fronteras de éste, es decir, los turistas que se desplazan en el interior del país lo hacen por motivos vacacionales, recreación, congresos y convenciones, trabajo y/o negocios, etc” (Portugal, 2008).

\section{Ecoturismo}

El ecoturísmo es según Diamantis y Ladkin en el año 1999, "el resultado directo de la aceptación mundial a favor de prácticas productivas más sostenibles y con menor impacto al medio ambiente", sin embargo Jost Krippendorf autor quien se considera pionero en este concepto, hace una fuerte crítica al turismo de masas bajo el argumento de su potencial destructor en el medio ambiente y economía dentro de las comunidades receptoras, reconociendo la necesidad de buscar una nueva opción (Rebollo, 2012).

El ecoturismo tiene como función proteger y cuidar el ambiente, con la intención de no manipular a la naturaleza, 
sino contemplar y admirar su belleza. Conocer a quienes han habitado por siempre en estos ecosistemas, desde las culturas ancestrales, hasta su flora las cualidades curativas y su fauna para respetar el lugar; por lo regular los centros ecoturísticos se encuentran en áreas ecológicamente protegidas. (Rebollo, 2012). De igual manera busca romper con la idea de que "lo legítimamente turístico se encuentra únicamente en grandes cadenas de hoteles, centros de diversión, restaurantes y por último que el espacio natural debe ser transformado a conveniencia de ser humano (Melo, 2013).

\section{Atractivo turístico}

Como una de las primeras definiciones encontramos a Zimmermann en 1957, quien comenta que "los atractivos turísticos son recursos turísticos que cuentan con las condiciones necesarias para ser visitados y disfrutados por el turista, es decir, que cuentan con planta turística, medios de transporte, servicios complementarios e infraestructura básica. Los atractivos turísticos deben considerarse como recursos pues han generado una actividad económica y representan divisas en la economía de un país" (Castellanos, 2015).

Por su parte Acerenza en 1984, identifica "los atractivos turísticos como el componente más importante del producto turístico, por cuanto son los que determinan la selección, por parte del turista, del punto del destino de su viaje, y son los que generan por tanto, una corriente turística hacia su localización y apunta asimismo que constituyen el principal motivo para que el turista lo visite el destino y son capaces de satisfacer las motivaciones primarias de viaje de los turistas" (Navarro, 2015). Por último, el diccionario de turismo, hotelería y transporte, nos ofrece una definición de atractivo turístico, la cual dice que "considera que se trata de un objeto o acontecimiento capaz de motivar a un turista a abandonar su domicilio habitual para trasladarse a conocerlo" (Wallingre \& Toyos, 2010).

\section{Marca cuidad o City marketing}

"El termino City Marketing ha sido mal entendido en muchas ocasiones, reduciéndose a una actividad de mera promoción a través de la cual mostrar una ciudad, principalmente de cara al turismo, apoyándose en discursos más o menos recurrentes: folletos con fotos de los monumentos principales, trajes regionales, gastronomía local y programa de fiestas. Pero de lo que estamos hablando es de una concepción mucho más amplia y potente. Se trata de trasladar el enfoque y las herramientas de marketing que aplicamos a una empresa o producto, a la ciudad, como un instrumento imprescindible en el desarrollo de sus estrategias y de su competitividad.” (Romero, 2009).

City marketing se concibe como la ciudad en el "Marketing como un producto, la cual se enfrenta a un mercado competitivo formado por miles de municipios y en donde convergen millones de agentes, además orienta su actividad hacia los ciudadanos del municipio los visitantes, los turistas y los inversores" esto según (Betancourt, 2008), sin embargo, se conoce que el termino de City marketing se introdujo en la literatura europea en los años '80. El City Marketing para Kotler, Haider y Rein en el año de 1993 se constituye como "una estrategia de desarrollo de la ciudad orientada a satisfacer, mejor que otras ciudades competidoras, las necesidades de los usuarios actuales y futuros del conjunto de los servicios de la ciudad” (Heredero, 2015).

Otros autores mencionan que "la mundialización y la extensión de la competencia: la puesta de valor de lo local: la rápida evolución de las herramientas de la comunicación y finalmente la evolución del marketing son factores que sin duda determinan el marketing urbano o el marketing de cuidad" (Benko, 2000). También se podemos encontrar otra definición a cerca del city marketing y se define como "El marketing de ciudad (city marketing) se puede definir como política activa de actuaciones orientadas, por una parte, a identificar y determinar las necesidades de sus diferentes públicos, reales y potenciales; y otra parte a desarrollar una serie de productos y servicios en la ciudad para satisfacer dichas necesidades, creando y potenciado su demanda" (Rojano \& Castilla, 2007).

\section{MARCO TEÓRICO}

\section{Turismo}

Se dice que "el turismo viene a diversificar la economía aportando divisas necesarias para impulsar el desarrollo económico, pero además los defensores del desarrollo de la actividad turística argumentan que el turismo no solamente genera divisas, sino que también alivia un poco el problema del desempleo y a largo plazo puede suministrar un sustituto de exportaciones tradicionales 
cuyo fruto es más inseguro que el turismo" (Portugal, 2008). Pero siempre se tiene que tener en cuenta la demanda turística la cual la "conforman turistas, viajeros y visitantes, tras este término se encuentra un grupo heterogéneo de personas, un agregado de personalidades con diferentes características e intereses tales como: sociales, económicos, recreativas" (Portugal, 2008), en estas demandas se encuentra un grupo denominado turismo de minorías los cuales se caracterizan por "viajar de forma individual, familiar y en grupo, sus núcleos elegidos son poco frecuentados turísticamente y lo hacen en cualquier época del año porque sus posibilidades económicas se lo permiten”(Portugal, 2008).

Si bien es cierto que existen muchos tipos de demanda, este segmento "prefiere que el consumidor de este tipo de servicio, podría dar una mejor idea de las actividades y productos que debemos ofrecer. No sin antes aclarar que, para cada actividad o especialidad en particular, hay un segmento de mercado con características propias." (Rogel, Rojas \& Ortega, 2011), es decir que cada segmento tenga bien especificado su perfil. Sin duda la demanda es importante, pero de igual manera es importante decir que los viajes tienen "como fin el realizar actividades recreati- vas de apreciación y conocimiento de la naturaleza a través del contacto con la misma, estas actividades pueden ser observación de astros, observación de flora y fauna, senderismo, educación ambiental, investigación biológica, observación de atractivos y fenómenos especiales de la naturaleza, y observación de atractivos naturales entre otros." (Rogel, Rojas \& Ortega, 2011).

Como recursos naturales SECTUR en el año del 2005 nos menciona que "los recursos naturales o culturales son el medio ambiente, la riqueza arqueológica y las expresiones históricas de tradición, estas se consideran la base del producto turístico, para que este recurso se convierta en atractivo turístico el hombre debe incorporar instalaciones equipamiento y servicios, de esta forma agrega valor en términos económicos" (Portugal, 2008). Pero como saber si un lugar tiene atractivo turístico o no, en este sentido, el "CICATUR-OEA (Centro Interamericano de Capacitación Turística de la Organización de Estados Americanos) propuso en 1974 una escala de valoración de recursos turísticos, que rápidamente se extendió en Latinoamérica y de uso prácticamente excluyente hasta la actualidad, como se muestra en la Tabla 3" (Navarro, 2015).

\section{Tabla N³ 3. Jerarquización de recursos turísticos.}

\begin{tabular}{ll}
\hline Jerarquía 5 & «Atractivo excepcional y de gran significación para el mercado turístico internacional, capaz por sí \\
& solo de motivar una importante corriente de visitantes (actual o potencial), cuantificada como un \\
& porcentaje del total de la demanda del país, o de algún mercado específico». Equivalente a recurso \\
& único en el mundo.
\end{tabular}

Jerarquía 4 «Atractivo con rasgos excepcionales en un país, capaz de motivar una corriente (actual o potencial) de visitantes del mercado interno o externo, pero en menor porcentaje que los de jerarquía 5 , ya sea por sí solo o en conjunto con otros atractivos contiguos». Equivalente a recurso único en el país.

Jerarquía 3 «Atractivo con algún rasgo llamativo, capaz de interesar a visitantes de larga distancia, ya sea del mercado interno o externo, que hubiesen llegado a su zona por otras motivaciones turísticas; o de motivar corrientes turísticas locales (actuales y potenciales)». Equivalente a recurso único en una jurisdicción subnacional.

Jerarquía 2 «Atractivos con méritos suficientes como para ser considerados importantes para el mercado interno, pero sin condiciones para el turismo receptivo y que forman parte del patrimonio turístico». Equivalente a recurso único en una localidad.

Jerarquía 1 «Atractivos sin méritos suficientes como para considerarlos en las jerarquías anteriores, pero que igualmente forman parte del parte del patrimonio turístico como elementos que pueden completar a otros de mayor jerarquía en el desarrollo y funcionamiento de cualquiera de las unidades que integran el espacio turístico». Equivalente a recurso complementario.

Jerarquía 0 «Atractivos cuyas cualidades no permiten incorporarlos a la jerarquía 1, que es el umbral mínimo de jerarquización». Equivalente a recurso sin características relevantes (no es un recurso turístico).

Fuente: Navarro, 20015. Recursos turísticos y atractivos turísticos: CONCEPTUALIZACIÓN, CLASIFICACIÓN Y VALORACIÓN. Cuadernos del turismo. Recuperado de http://www.redalyc.org/pdf/398/39838701014.pdf 
De igual manera existen otras formas de clasificar un atractivo turístico, puesto que otros autores dicen que se "debe analizar los recursos tangible e intangible, que son susceptibles de aprovechamiento turístico y considerar el potencial de atracción, que pueda motivar un desplazamiento no lucrativo; de igual manera la accesibilidad de llegar, la señalización con guías turísticas, folletos, señales urbanas, el nivel de afluencia, el nivel de uso, los elementos de valoración y las actividades actuales o posibles relacionadas con el recurso del lugar” (Navarro, 2015).

Sin embargo, también se puede llevar a cabo con los siguientes criterios jerárquicos, "primero los que no tienen mérito suficiente pero que pueden complementar a otros de mayor jerarquía: luego los que cuentan con algún rasgo llamativo y motivan a quienes llegan al lugar por otros atractivos, después los que tienen rasgos excepcionales con capacidad de motivar demanda nacional, ya sea por si solos o en conjun- to con otros atractivos; y por último los que poseen rasgos excepcionales y de gran significación para el mercado internacional capaces por si solos de motivar el desplazamiento turístico de esa demanda” (Wallingre, 2011).

\section{Ecoturismo}

El ecoturismo es quizás la palabra que comercialmente ha tenido más éxito en el desarrollo y mercadeo de una actividad turística novedosa, asociada al aprovechamiento de los recursos naturales y culturales de una región. Por su éxito comercial, también se ha prestado para un empleo indiscriminado que ha obligado a crear nuevas terminologías, que permitan diferenciar el producto y garantizar de algún modo su calidad (Báez\&Acuña, 1998). Para Latinoamérica, se presentan a continuación algunos acontecimientos que refieren a los antecedentes generales de la adopción del ecoturismo en los países latinoamericanos (Tabla 4).

\section{Tabla $N^{\circ} 4$. Antecedentes generales del ecoturismo en Latinoamérica.}

\begin{tabular}{ll}
\hline Fecha y sede & Acontecimiento \\
\hline Diciembre de 1974 & Primera Reunión Centro- \\
San José, Costa Rica & americana sobre Manejo \\
& de Recursos Naturales y \\
& Culturales
\end{tabular}

1974 Coyococ, México

Octubre de 1975 El Salvador

1986 Caracas, Venezuela

1987 Guatemala

1988 Chile

1991 México

1992 Guadalajara, México
Seminario sobre Modelos de Utilización de Recursos Naturales, Medio Ambiente y Estrategias de Desarrollo

IV Reunión de Ministros de Agricultura de Centroamérica

Taller sobre planificación de sistemas nacionales de áreas silvestres protegidas

Segunda Reunión Centroamericana sobre Manejo de Recursos Naturales y

Culturales

Taller Internacional sobre Interpretación en Áreas Silvestres Protegidas Taller Internacional sobre manejo de las reservas de la biósfera

Congreso Iberoamericano de Educación Ambiental en América Latina

\section{Acuerdos y logros alcanzados}

Se propuso la creación de un sistema de parques y reservas en zonas de gran diversidad ambiental y cultural y, con alto potencial turístico. También se planteó el poner en marcha un comité regional para la conservación de los recursos y adecuar la legislación para su manejo eficiente (CATIE, 1989).

En este seminario se determinaron modelos alternativos de desarroIlo socioeconómico que contemplan las características ambientales y culturales de cada región con la finalidad de disminuir la inequidad que generan los modos de consumo y producción actuales (González, 2001).

Con base en esta reunión se estableció como prioritario la conservación, restauración y tratamiento de los recursos naturales por ser la base para el bienestar y el desarrollo local (CATIE, 1978).

Se acordó que en Latinoamérica se debe contar con un sistema de áreas protegidas reconocido legalmente para que estas zonas se operen de manera eficiente (Cracco, 2006).

Tuvo por objetivo establecer estrategias para el fortalecimiento del sistema de áreas naturales protegidas y promover la conservación para el desarrollo local (CATIE, 1989).

Se abordaron aspectos generales sobre el manejo de las áreas protegidas en Latinoamérica (FAO, 1988).

A partir de este taller se recomendó la inclusión de componentes de evaluación y monitoreo en la gestión de las áreas de conservación (Cracco, 2006).

Se implementaron programas para formar especialistas ambientales y se impulsó la organización y comunicación entre educadores ambientalistas (Ruvalcaba, 2010). 
$\frac{\text { Fecha y sede }}{1992 \text { Caracas, Venezuela }}$

Octubre 1995 Quito, Ecuador

2009 Caracas, Venezuela

\section{Acontecimiento}

Turismo en áreas protegidas y IV Congreso Mundial de Parques Nacionales

Reunión para América Latina sobre la gestión de programas nacionales de educación y capacitación para el medio ambiente y el desarrollo

Primer Congreso Centroamericano de Ecoturismo

\section{Acuerdos y logros alcanzados}

Se reconoció que el turismo contribuye a la conservación y al desarrollo de las comunidades locales (Naciones Unidas, 2001), así como la importancia de los programas de seguimiento y monitoreo en la conservación ambiental (Cracco, 2006).

Con base en esta reunión se implementó la red de Educación Ambiental de la Unión Internacional para la Conservación de la Naturaleza (UICN)-Sur (Ruvalcaba, 2010).

El congreso propuso las siguientes recomendaciones: creación de consejos nacionales de ecoturismo, programas de educación en ecoturismo, reinversión para la conservación, cooperación entre organizaciones de turismo y políticas para el desarrollo del ecoturismo, principalmente (Naciones Unidas, 2001).

Fuente: Aragón, M. (2014), Análisis del ecoturismo como alternativa de desarrollo

Sustentable en Latinoamérica (México, Costa Rica y Ecuador). El Colegio de la Frontera Sur. Université de Sherbrooke.

\section{El Ecoturismo debe desarrollarse bajo los siguientes valores y que deben ser seguidos tanto por el turista como por la gente local:}

- $\quad$ Responsable con el uso y manejo de atractivos turísticos.

- $\quad$ Respetuoso de las comunidades donde se desarrollan las actividades.

- $\quad$ Honesto con el producto para que conserve sus condiciones auténticas mientras se presenta al turista.

- $\quad$ Educativo para que el visitante adquiera nuevos conocimientos del lugar visitado.

- Interactivo al permitir un contacto directo con los recursos naturales y culturales.

- Democrático para que los beneficios obtenidos se repartan de manera equitativa (Romero, 2008).

\section{Perfil del ecoturista (Báez, A. 1996):}

- $\quad$ Interesado en tener contacto directo con la naturaleza.

- Interesado en conocer diferentes formas de entender y vivir la vida (que busca un intercambio cultural).

- $\quad$ Dispuesto a aprender, siempre activo y dinámico.

- Generalmente educado y con algún conocimiento previo sobre el destino, el recurso a visitar y las posibles actividades a realizar.

- Cuidadoso de su condición física y anímica.

- $\quad$ Prefiere el contacto directo con las personas y busca establecer lazos de amistad

- $\quad$ Prefiere un servicio personalizado y con sello de calidad.

- Está anuente a colaborar con iniciativas para un mejor manejo de desechos, reducción del consumo de agua y energía y cualquier otro esfuerzo para disminuir el impacto negativo.

A cualquier actividad ecoturística, ya sea a nivel nacional, regional o local, es indispensable levantar inventarios de los atractivos ecoturísticos, tanto existentes como potenciales, puesto que el inventario nacional de atractivos ecoturísticos no es más que la suma de los diferentes inventarios ecoturísticos regionales de un país (Lascuráin, 1998).
Como parte del inventario de recursos naturales se encuentran lagos, ríos, playas, montañas, valles y praderas. De ellos no sólo nos permiten extraer alimentos y materia prima, sino que también son admirables por su gran belleza, por lo tanto, estos recursos naturales son considerados también como inventario turístico (Rebollo, 2012). 
Es posible identificar áreas y atracciones naturales como los elementos más importantes del sistema turístico que motivan a la visita y que ofrecen al turista experiencias y memorias únicas, porque primero están los recursos naturales y los componentes de su medio ambiente. Lagos, lagunas, montañas, valles, desiertos, selvas, planicies, playas, esteros, colinas, sierras, cañadas, montes nevados y en segundo lugar cualquier destino es caracterizado principalmente por su tipo de ecosistema, es decir la naturaleza y apariencia de su paisaje y su clima, como el tipo de condiciones de frio de calor, humedad, vientos, altura y características específicas dadas por su altitud y localización en el hemisferio (Rebollo, 2012). Se pueden clasificar los atractivos ecoturísticos de un área en tres categorías básicas:

- Atractivos focales: Los cuales de un área o región determinada siempre se referirán a los elementos distintivos de patrimonio natural y/o cultural que se encuentran en dicha área. Son aquellos rasgos intrínsecos de singularidad que mejor caracterizan a dicho sitio o región y el motivo fundamental por lo cual los ecoturistas querrán visitarlo. Algunas áreas protegidas existen debido a un recurso muy especial o determinado que poseen y por lo tanto, constituye asimismo su principal atractivo focal.

- $\quad$ Atractivos complementarios: También se refieren a elementos de patrimonio natural y/o cultural que se encuentran en un área determinada, pero que no poseen el grado de importancia o singularidad en cuando a atracción turística de los atractivos focales. Es decir, por sí solos quizá no ejercerían suficiente atractivo para motivar a un ecoturista o desplazarse a ese sitio, sin embargo, constituyen motivados de interés adicional y valor agregado para el ecoturista, contribuyendo a una experiencia turística de mayor riqueza y diversidad, al indicar al visitante a que permanezca mayor tiempo en el área en cuestión y al ofrecerle la posibilidad de actividades adicionales. Los atractivos ecoturísticos complementarios también pueden contribuir a evitar concentraciones excesivas de turistas en un solo lugar $\mathrm{y}$, al mismo tiempo, a propicias al desplazamiento de los visitantes por diversos sitios del área correspondiente.

- $\quad$ Atractivos de apoyo: Estos están constituidos por los elementos artificiales ya sea en instalaciones o servicios, que proporcionan al visitante diferentes satisfactores. Aquí se incluyen los alojamientos, restauran- tes, centro de interpretación, senderos y miradores, servicios de paseos a caballo o en lancha, etcétera. Los atractivos de apoyo siempre se agregan a posteriori, para dar soporte a los atractivos focales y complementarios que ya existen, por naturaleza propia, en un destino ecoturístico determinado (Lascuráin, 1998).

Para el turista medio, la mera contemplación en un área protegida de enormes concentraciones de aves acuáticas, constituye de por sí un atractivo del más alto nivel. Aunque no conozca el nombre específico de todas las aves, la enorme concentración de éstas y la forma o el color distinto de algunas de ellas bastarán para causar una honda impresión en cualquier visitante con un mínimo de sensibilidad. Sin embargo, los verdaderos ecoturistas, tanto nacionales como extranjeros, desearán conocer algo más de las regiones que visitan: la estructura de sus ecosistemas, su origen y evolución geológica, las principales interrelaciones ecológicas que ahí se dan, los elementos culturales regionales, su entorno geográfico y los nombres de las plantas y animales más característicos o llamativos que ahí se encuentran, es decir, de las especies focales (Lascuráin, 1998).

Uno de los primeros estudios formales que se hicieron acerca de la situación del ecoturismo en México fue el texto de Ceballos-Lascuráin en el año 1994 llamado Estrategia Nacional de Ecoturismo. En él, se hace un diagnóstico completo del estado que guardaba esta actividad en el contexto mexicano, se describían las ventajas competitivas y se señalaba la necesidad de llevar a cabo más investigación acerca de los impactos económicos de esta actividad. También se presenta un plan de acción el cual nos menciona diferentes partes como que la creación de mecanismos para la cooperación inter-institucional, la creación de un inventario de productos ecoturísticos, la creación de un plan estratégico inclusivo, el diseño de manuales de manejo de impactos ambientales y culturales, capacidad de carga, entrenamiento, promoción y el establecimiento de mecanismos de autofinanciamiento dirigidos principalmente a la conservación del medio ambiente.

Lascurain concluye que el ecoturismo en México estaba en sus primeros pasos y que siguiendo un plan de acción aseguraría un desarrollo orientado y bien organizado (Guerrero, 2010). Sin embargo, Rodríguez en el 2010 en su artículo comenta que el ecoturismo en México es, solo una promesa. Sin duda alguna el ecoturismo puede convertirse en una herramienta poderosa de desarrollo en 
cualquier latitud, sin embargo, para el caso de México la evidencia sugiere que esto todavía no es así y que el panorama no es muy favorable para que esta situación se revierta. La agenda política de México debe contemplar la creación de condiciones necesarias para contrarrestar lo anterior, a fin de que actividades como el ecoturismo, puedan crecer y desarrollarse de manera positiva.

El ecoturismo promueve la integración del turista con la comunidad local a través de paseos guiados en zonas de ecosistemas frágiles; tal como selvas tropicales o zonas naturales protegidas. De esta manera se supervisa el posible 20 daño ambiental y es posible evitarlo o minimizarlo. A través del ecoturismo es posible también enmendar daños ambientales ocasionados con anterioridad (Rebollo, 2012).

\section{El city marketing}

La imagen de una ciudad es la síntesis de su identidad, que está definida por la suma de atributos de carácter permanente, que componen su esencia y sirven para diferenciarla de otras urbes. Por lo tanto, la identidad de la ciudad tiene una doble dimensión: funcional y cultural. La dimensión funcional se concreta en su propósito estratégico, entendido éste como su razón de ser, y se expresa mediante su modelo de ciudad, que no es más que la estrategia de la misma para cumplir su objetivo principal a medio y largo plazo, a partir del conjunto de políticas funcionales y formales. La cultura corporativa, otra dimensión de la imagen de la identidad, está compuesta por los valores compartidos por el conjunto de los ciudadanos y las presunciones de éstos sobre su ciudad, del entorno y de todo aquello que pueda llegar a influir en ella (Betancourt, 2008).

La proyección de la imagen de una ciudad, se da a partir de un imaginario, que considera los hitos arquitectónicos como referentes "tangibles" obligados para sus habitantes como para los visitantes, además de múltiples factores culturales que contribuyen a formar un "intangible", el cual afecta sensiblemente la percepción y elección de productos, servicios y destinos turístico. Según la visión de Mea Della éste se construye a través de un complejo proceso de influencias, en el que intervienen múltiples factores de distinto orden:

- Los naturales: Clima, atractivos geográficos y suelo.

- Los económicos: Nivel de desarrollo, actividades, productos y marcas locales, servicios, corporaciones y tecnología.
- Los políticos: Sistema de gobierno y desarrollo institucional.

- Los geopolíticos: Vínculo histórico con ciudades y departamentos.

- Los socioculturales: Población, nivel educativo, expresiones culturales y calidad de vida (Mancilla, Morales \& Lugo, 2012).

El city marketing está enfocado en tres aspectos básicos:

- Para inversionistas: Es el cual busca atraer inversionistas y empresas extranjeras, para la creación y/o expansión de nuevos negocios, con la intención de traer consigo desarrollo a nivel económico y que se vea reflejado en aumento de capitales y fuentes de empleo.

- Para residentes: Es el cual busca de alguna manera atraer nuevos residentes a las ciudades, buscan aumentar su población productiva por intermedio de atractivos programas para obtención de visas de residente permanente.

- Para visitantes: Es el cual busca atraer visitantes temporales, para incentivar el turismo y al mismo tiempo atraer capitales importantes que incentiven diferentes sectores turísticos.

Específicamente, el City marketing desempeña cuatro funciones básicas:

- Lograr una combinación óptima de las características y los servicios de la ciudad, desde el punto de vista de los residentes, visitantes e inversores.

- Articular una oferta de incentivos que aumente el atractivo de la ciudad para los actuales y futuros usuarios de sus servicios.

- Asegurar un rápido y eficiente acceso de la ciudad a los mercados de interés.

- Transmitir al público objetivo la imagen y las ventajas comparativas de la ciudad.

Sin duda alguna la gestión de marca se ha convertido en uno de los temas obligados para los Gobiernos y las organizaciones privadas y públicas de las ciudades y de los países. Como lo son "los naturales: clima, atractivos geográficos, suelo; los económicos: nivel de desarrollo, actividades, productos y marcas locales, servicios, corporaciones, tecnología; los políticos: sistema de gobierno, desarrollo institucional; los geopolíticos: vínculo histórico con ciudades, departamentos; los socioculturales: población, nivel educativo, expresiones culturales, calidad de 
vida. De nuevo frente de gestión cobra gran importancia, ya que gracias a su planificación se pueden evidenciar logros importantes en el fortalecimiento de la competitividad y la imagen de las ciudades y de los países" (Martínez, 2007).

Daniel Ivoskus nos dice que "la marca no es un producto y hay que mantener esta afirmación al crear una marca ciudad, la cual se reconocerá en un determinado territorio. Muchos autores han intentado clasificar la ciudad, ya sea por la población, desde la geografía, por su madurez, a través de criterios cualitativos como la especialización de la misma, por la seguridad, el flujo financiero, etc. (Marín, 2010), y Pablo Lezama nos da algunos ejemplos de marca cuidad como "el trabajo sobre la marca Barcelona, el famoso "I love NY", Milán o la ciudad Armani, la Patagonia Argentina, Los Angeles y Hollywood; y además de lugares, barrios tales como: Tribeca y Harlem en Nueva York; Palermo Soho, Barracas dulce y San Telmo Gay en Buenos Aires. Todos estos son algunos de los ejemplos del posicionamiento que se eligió construir para diferenciarse de otros lugares del mundo" (Marín, 2010).

\section{La marca Guadalajara}

El gobierno de Zapopan realizó un presentación de la marca Guadalajara Guadalajara en donde el ex Gobernador de Guadalajara Enrique Alfaro menciona que "la marca cuidad es un referente para evitar la pérdida de su identidad", de igual manera comentó que "la marca cuidad es un elemento de identidad, es una herramienta de promoción y de posicionamiento de Guadalajara que desde hacía muchos años la cuidad necesitaba, que el sector privado había pedido y que ahora existe gracias a la voluntad de construir una política de acuerdos" (Gobierno de Zapopan, 2016). En la presentación de la marca también participó el Gobernador del estado de Jalisco, Aristóteles Sandoval, quien mencionó que "los nombres de las ciudades son marcas, son referencia sobre una identidad".

Se han convertido en activos estratégicos. Tener una marca cuidad fuerte sirve para identificar, cohesionar re ilusionar a la ciudadanía. Sirve para proyectarnos internacionalmente, captar proyectos e inversionistas" (Gobierno de Zapopan, 2016). Cabe destacar que también Pablo Lemus realizó algunos comentarios los cuales dicen "somos una sola voz, hoy quedó demostrado que nuestra identidad es ser tapatío. Es un orgullo pertenecer a esta ciudad, una marca que tendrá grandes beneficios para todos. Esto es un hecho histórico que le da voz a \#GuadalajaraGuadalajara, felicidades a todos por que hoy se presenta su marca, nuestra marca” (Gobierno de Zapopan, 2016).

Guadalajara es la segunda ciudad más poblada de México, después de la ciudad de México, con este ejercicio de identidad se pretende promocionar y posicionar la metrópoli internacionalmente, incrementar la afluencia turística y continuar atrayendo inversiones del extranjero (García, 2016). Los creadores de la marca de Guadalajara Guadalajara, mencionan que se aprovechó la gran oportunidad de construir la primera marca sonora en el mundo, puesto que la marca está basada en la canción compuesta por Pepe Guízar, la cual representa un símbolo natural de la cuidad que refuerza y provoca sensaciones, emociones e ideas ya posicionadas. "La canción tiene los ritmos característicos del mariachi y su letra retoma elementos esenciales de la identidad de la ciudad y de la región, condensándolos en una sola frase: Guadalajara Guadalajara, la cual se lee cantando, puesto que ya está posicionada a nivel internacional” (Valenzuela, 2016).

Se ha creado un logotipo muy expresivo, con tipografías que varían de tamaño y que nos recuerda a los códigos usados en los cómics, donde se intenta plasmar en el propio logo la musicalidad de este famoso estribillo, como ya mencionamos anteriormente, la tipografía es la Chinese rocks regular, la cual se usa para darle un toque rustico que pretende poner valor la herencia de un pasado preindustrial, donde los comercios o incluso carteles hacían gala de un diseño manual y mucho más estandarizado. La marca presenta además tres colores principales y alternantes: magenta, naranja y cian, que recalcan el carácter colorista del folclore mexicano (García, 2016).

Se trabajó una reinterpretación de la canción, en la que se mezcló el mariachi con música tradicional, elementos de rock, electrónica y jazz, también colaboraron artistas como Venado Azul, un grupo wixarika de música regional; Telefunka, un grupo de música electro-acústica; Cuca representando el rock pesado, disruptivo; Paco Padilla o el Mariachi Viva Xalisco representando lo tradicional de la música mexicana, con; Sara Valenzuela y Abigail Vázquez, destacadas exponentes del jazz; Mike Laure Jr. con música popular; además, Cecilia Toussaint, Celso Piña, Alfonso André, "el Vampiro", Pato Machete, Dr. Shenka con el propósito de integrar a todo México (Valenzuela, 2016),

En las agendas de competitividad de los destinos turísti- 
cos de México, se menciona que éste no ha sabido aprovechar el total de beneficios que brinda la actividad turística, un claro ejemplo de esto es la ciudad de Guadalajara la cual es uno de los estados emblemáticos de México, esta cuidad es destacada por su gran aportación cultural, tradición y edificaciones históricas, sin embargo, no ha sabido aprovechar, el patrimonio natural que tiene para darle un uso recreativo o turístico, puesto que cuenta con un gran potencial en este sector. Ahí entramos a uno de los atractivos el cual es la barranca de río Santiago (SECTUR, 2014).

\section{La Barranca del Río Santiago}

La Barranca del Río Santiago es un elemento natural de gran valor, dado su nivel de paisajismo, puesto que cuenta con variedad de recursos con potencial turístico que no se han aprovechado convenientemente para un uso recreativo y turístico. Además del paisaje, en la barranca se encuentran una importante cantidad y variedad de recursos con potencial turístico que hasta ahora no han sido aprovechados convenientemente para su uso recreativo y turístico (SECTUR, 2014). Esta barranca es un recurso de tal importancia, que se han creado instrumentos legales para su protección y conservación. El municipio de Guadalajara decretó el área como Zona Sujeta a Conservación Ecológica, mientras que el municipio de Zapopan le otorgó la categoría de Área Municipal de Protección Hidrológica.

A nivel federal existe una propuesta de decretar la Barranca del Río Santiago, junto la Barranca del Río Verde, como un Área Natural Protegida (ANP), sin que hasta ahora se formalice su instrumentación legal (SECTUR, 2014). Sin embargo, es necesario destacar la problemática ambiental presente en la barranca, ya que es uno de los ríos más contaminados de México debido a las descargas domésticas e industriales las cuales en su mayoría no son tratadas. Esta situación le resta potencial al río Santiago que, en su contexto natural, bien podría ser aprovechado con fines recreativos. A pesar de la condición del río, la barranca sigue teniendo un alto potencial de aprovechamiento turístico y recreativo, pero para ello necesitan implementarse políticas públicas encaminadas al saneamiento integral de la cuenca del Río Santiago, así como la implementación de proyectos productivos y turísticos que detonen el desarrollo local de la zona (SECTUR, 2014).

Un hecho geográfico de gran envergadura es el realizado por el río Santiago sobre lo que ahora es la Barranca que lleva su nombre, dando lugar a una depresión que en su parte más erosionada puede alcanzar los 500 metros de profundidad aproximadamente, esto según el INEGI en el 2010 (SECTUR, 2014). Este afloramiento geológico alberga un bosque de galería que contiene diversas especies de fauna, pero sobre todo de flora, algunas de ellas incluso son catalogadas como endémicas, situación que vuelve aún más atractiva a la Barranca del río Santiago para ser estudiada y conservada. La problemática de este elemento geográfico es la contaminación del río Santiago, el cual arrastra todos los desechos domésticos e industriales de la Cuenca Lerma- Chapala-Santiago; sin embargo, su principal problema es la falta de reconocimiento y valoración por parte de los habitantes locales (SECTUR, 2014). La Barraca del Río Santiago es un escenario natural con gran potencial de aprovechamiento turístico.

En la década de los '70 se proyectó un complejo turístico para la Barranca del Río Santiago correspondiente al municipio de Zapopan, en éste se contemplaba la instalación de uno o varios funiculares, teleférico, jardín botánico, restaurante, bungalows, etc., en las instalaciones del Parque Mirador Dr. Atl., no obstante, sólo quedó en propuesta. En el año 2005 Guadalajara ganó la sede para albergar el Museo Guggenheim, el cual se ubicaría en los terrenos aledaños al Parque Mirador Independencia en la Barranca del Río Santiago. En el 2009 el proyecto fue cancelado debido a la falta de presupuesto. En diciembre de 2009 se anunció el proyecto "Barranca Museo de Arte Moderno", el cual vino a sustituir al Guggenheim.

El proyecto tiene un costo aproximado de 30 millones de dólares y para 2013 lleva un avance del $80 \%$ en su estructura. Actualmente se tiene pensado hacer un teleférico, es posible incluso con tecnología mexicana, como el Transporte Urbano Elevado Personalizado (TUEP), propuesto en la Ciudad de México, con el apoyo del Consorcio Dina. También se propone rescatar la infraestructura existente para instalar un funicular que vaya de Huentitán hacia el fondo de la Barranca, en la localidad Ilamada Las Juntas, en el municipio de Guadalajara y se contempla un costo de 320 millones de pesos, mismos que serían invertidos en un período de tres años aproximadamente (SECTUR, 2014). Según Martínez en 1984, la Barranca es un impresionante murallón quebrado de tramo en tramo, por los boquetes que en su vertiente norte han abierto los ríos que aportan sus aguas al Santiago. El panorama que se advierte frente a Huentitán, La Experiencia y San Cristóbal, es el de una cortina de roca cuyos pliegues casi horizontales compusieron, hace millones de años el fuego y el agua. El Santiago, "incansable zapador de 
la Barranca", espejea ostentoso para asombro de paseantes y turistas, análisis y estudio de geólogos y quebradero de cabeza de constructores de puentes, vías férreas, malaca- tes y carreteras (SECTUR, 2014). Los recursos potenciales en que pueden hacer mejor y crear actividades recreativas son presentados en la tabla 5.

Tabla N 5. Recursos con potencial turístico en la Barranca del Río Santiago (ZMG).

\begin{tabular}{|c|c|c|c|}
\hline Recurso & Sitios & Ubicación & Observaciones \\
\hline \multirow{4}{*}{ Cascadas } & Las 7 cascadas & Tonalá & Sólo en temporada de lluvias \\
\hline & Cola de Caballo & Zapopan & Dentro del Parque Mirador Dr. Atl \\
\hline & La Soledad & Zapopan & Sobre cauce del Arroyo La Soledad \\
\hline & San Lorenzo & Zapopan & Sobre cauce del Arroyo Milpillas \\
\hline \multirow{3}{*}{ Miradores } & Huentitán & Guadalajara & Barranca de Huentitán \\
\hline & $\begin{array}{l}\text { Parque Mirador } \\
\text { Independencia Dr. Atl }\end{array}$ & Guadalajara & $\begin{array}{l}\text { En este lugar se está edificando el } \\
\text { Museo de Arte Moderno y } \\
\text { Contemporáneo de Guadalajara } \\
\text { (Museo Barranca) }\end{array}$ \\
\hline & Parque Mirador Dr. Atl & Zapopan & $\begin{array}{l}\text { Se encuentra bastante deteriorado, } \\
\text { sin atención del gobierno municipal }\end{array}$ \\
\hline \multirow{3}{*}{$\begin{array}{l}\text { Cuerpos de } \\
\text { agua }\end{array}$} & Arroyo La Soledad & Zapopan & $\begin{array}{l}\text { Tributario más limpio del Río } \\
\text { Santiago }\end{array}$ \\
\hline & Arroyo Milpillas & Zapopan & \\
\hline & $\begin{array}{l}\text { Géiseres de La } \\
\text { Soledad }\end{array}$ & Zapopan & $\begin{array}{l}\text { Localizados sobre el cauce del } \\
\text { Arroyo La Soledad }\end{array}$ \\
\hline \multirow[b]{2}{*}{ Cañón } & Cañón Azul Turquesa & Zapopan & $\begin{array}{l}\text { Es el cañón formado por el Arroyo } \\
\text { La Soledad }\end{array}$ \\
\hline & $\begin{array}{l}\text { Cañón del Río } \\
\text { Santiago }\end{array}$ & $\begin{array}{c}\text { Tonalá, } \\
\text { Guadalajara y } \\
\text { Zapopan } \\
\end{array}$ & $\begin{array}{l}\text { Accidente geográfico de alto valor } \\
\text { paisajístico y medio ambiental para } \\
\text { la ZMG }\end{array}$ \\
\hline Elevaciones & $\begin{array}{l}\text { Peñón de Los } \\
\text { Camachos }\end{array}$ & Zapopan & $\begin{array}{l}\text { Desde aquí es posible observar una } \\
\text { panorámica de la barranca }\end{array}$ \\
\hline Cavernas & $\begin{array}{l}\text { Grutas de Los } \\
\text { Camachos } \\
\end{array}$ & Zapopan & Comentarios \\
\hline \multirow{3}{*}{ Balnearios } & Los Camachos * & Zapopan & Primer balneario de Guadalajara \\
\hline & San José * & Zapopan & \\
\hline & Huaxtla * & Zapopan & Negocio comunitario \\
\hline \multirow{3}{*}{$\begin{array}{l}\text { Rutas } \\
\text { (caminata, } \\
\text { Trekking, } \\
\text { senderismo) } \\
\end{array}$} & Huentitán & Guadalajara & Barranca de Huentitán \\
\hline & Oblatos & Guadalajara & Barranca de Oblatos \\
\hline & $\begin{array}{l}\text { Ixcatán - La Soledad - } \\
\text { Huaxtla }\end{array}$ & Zapopan & También como ruta ciclista \\
\hline \multirow{4}{*}{$\begin{array}{l}\text { Manifestacion } \\
\text { es culturales e } \\
\text { históricas }\end{array}$} & Puente de Arcediano & Guadalajara & Primer puente colgante de México \\
\hline & $\begin{array}{l}\text { Fiesta de los } \\
\text { Tastoanes }\end{array}$ & Zapopan & En la localidad de Ixcatán \\
\hline & Templo de Ixcatán & Zapopan & $\begin{array}{l}\text { Construcción franciscana que data } \\
\text { de } 1580\end{array}$ \\
\hline & $\begin{array}{l}\text { Templo de San } \\
\text { Esteban }\end{array}$ & Zapopan & $\begin{array}{l}\text { Construcción franciscana que inicia } \\
\text { su construcción en } 1691 \text { y se } \\
\text { terminó en } 1726\end{array}$ \\
\hline
\end{tabular}

Fuente: SECTUR (2014). Agendas de competitividad de los destinos turísticos de México. Secturjal. Recuperado de https://secturjal.jalisco.gob.mx/sites/secturjal.jalisco.gob.mx/files/u16/agenda_guadalajara.pdf 
Existen sitios para una aventura en Zapopan como lo es la página de Huaxtla.org sitio oficial de Aventura en Zapopan. Esta es una página sin fines de lucro que busca dar a sus visitantes información sobre los maravillosos recorridos en contacto con la naturaleza con que cuenta Jalisco y en particular el municipio de Zapopan, para conocer el Cañón de Huaxtla, El Bosque de la Primavera, El Cerro del Diente o cualquier otra Aventura dentro del municipio de Zapopan. (Huaxtla, s.f.).

\section{METODOLOGÍA}

La metodología de la investigación está conformado por un enfoque cuantitativo, ya que para la resolución de aportes, se tomaron en cuenta datos estadísticos de una base de datos que proporciono el gobierno del estado de Jalisco con la intención de dar resultados anuales del año 2016, los indicadores a rescatar fueron los siguientes: primero la motivación a los destinos turísticos, medio por el que se enteró, lo que más le gusto, opinión sobre el servicio, como consideran el atractivo natural, medio por el que arriban al destino y gasto promedio. Esto con el fin de determinar si al turismo nacional le interesan los factores de atractivos turísticos y ecoturísticos.

Tipo de estudio

El tipo de investigación es exploratoria que según Sampieri los estudios exploratorios se efectúan, normalmente, cuando el objetivo es examinar un tema o problema de investigación poco estudiado o que no ha sido abordado antes. Es decir, cuando la revisión de la literatura reveló que únicamente hay guías no investigadas e ideas vagamente relacionadas con el problema de estudio, los estudios exploratorios nos sirven para aumentar el grado de familiaridad con fenómenos relativamente desconocidos, en el caso de la investigación se encontró que hay poca, casi inexistente información sobre la Barranca del Río Santiago.

En la investigación también se utiliza el tipo descriptivo que según Sampieri selecciona una serie de cuestiones y se mide cada una de ellas independientemente, para así describir lo que se investiga, a lo largo de la investigación se describió atractivos turísticos, ecoturismo y city marketing. Cabe señalar que es una tesina documental, dado que todo se basó en información y no existe una muestra, ni población, ni encuestas.
Análisis de datos

En base a un estudio realizado por el gobierno del Estado de Jalisco, se tomaron los siguientes indicadores:

- Los medios de información por los cuales se enteran de un destino el $53.1 \%$ por recomendación, el $17.2 \%$ Internet, el 12.5 por que ya conocía el lugar, el 5.8\% agencia de viajes, el $3 \%$ por medios masivos y el $8.5 \%$ por otro medio.

- Lo que más les gusto al $28.8 \%$ son los atractivos turísticos, al $25 \%$ la hospitalidad, al $13.12 \%$ el destino, $8 \%$ actividades recreativas, $6.3 \%$ el comercio, al $5 \%$ los servicios turísticos, $4.1 \%$ la gastronomía, 3.1\% el clima y $2.5 \%$ templos.

- La opinión sobre el servicio es: El 89.9\% dijo que era excelente el trato personal y el $10.1 \%$ dijo que regular, la relación calidad precio, el 78\% dijo que excelente, $17 \%$ dijo regular y el $5 \%$ dijo malo: la limpieza el $88.3 \%$ dijo excelente, el $10.4 \%$ dijo regular y el $1.2 \%$ dijo malo.

- Diversos aspectos como atractivo natural: el $88.1 \%$ dijo excelente, $8.9 \%$ dijo regular y el $3 \%$ dijo malo; actividades de ocio el $90.1 \%$ dijo excelente, el 9.7 dijo regular y .2\% dijo malo. El medio de transporte que utilizan para llegar al destino es $46.1 \%$ automóvil y 53.1\% autobús.

\section{CONCLUSIONES}

Como conclusión de la investigación se menciona que la marca Guadalajara Guadalajara solo tiene un enfoque cultural e histórico, dejando atrás el aspecto ecoturístico, el cual según la secretaria de turismo existe un gran potencial, pues como se ha venido mencionando en las agendas de competitividad no se ha sabido aprovechar al máximo la Barranca del Río Santiago. A nivel nacional la cuidad marca Guadalajara está posicionada, gracias a que está basada en una canción muy famosa del compositor Pepe Guízar, la mayoría de los mexicanos la hemos escuchado alguna vez. Por lo que ampliar un enfoque de no solo ver la cuidad de manera arquitectónica, cultural y religiosa, se puede ampliar a otros sectores.

Bajo una perspectiva de turismo nacional y ecoturística la respuesta para nuestro problema de investigación es si pues la marca se puede ampliar hacia este sector con la 
intención de poder generar más turismo hacia ese tipo de lugares, junto con una ampliación de trabajo para la industria hotelera, realizar actividades recreativas que se cumplan las normativas de las instituciones correspondientes para no ocasionar el ya deteriorado ecosistema, sino que por el contrario se consiga un apoyo para poder limpiar la zona y tener un recurso más.

Sin embargo, nos encontramos con ciertas limitaciones para que cualquier proyecto de mejora se presente ya que la mayoría debe ser presentada ante la secretaria de turismo y que por las próximas elecciones del país cada vez se cambian las estrategias dependiendo a los nuevos proyectos de cada gobernador. Lo que ocasiona que los planes a largo plazo no sean factibles. De igual manera entra en conflicto ya que la marca Guadalajara está en el sector público, todavía no existe apoyo de parte del sector privado, por lo que es más complicado.

Propuestas

- Como primera propuesta está la realización de un mapa que señale el acceso y la ruta para llegar a los diferentes destinos de la Barranca, ya que, por el desconocimiento de muchos de los potenciales atractivos turísticos, es difícil el acceso a estos lugares, no están señalados, no se tiene una ruta estructurada.

- La segunda propuesta es creación de distintas tirolesas que sean atractivas para los diferentes públicos y edades de los visitantes. Como lo puede ser el de Huentitán a las Juntas.

- Tercera propuesta, que se tenga un control de contaminación, es decir, el tratamiento de aguas residuales de operación, un buen manejo de residuos sólidos y líquidos, la plantación y cuidado del medio ambiente o bien de los recursos naturales, con el fin de aumentar el atractivo turístico del lugar.

- Cuarta propuesta brindar apoyo a agencias de viajes para la elaboración de publicidad, en donde se les incentive a realizar cortos viajes a los diferentes atractivos con los que cuenta la Barranca del Río Santiago. Con el fin de aprovechar el lugar e impulsar el empleo en el sector turístico, la capacitación de los empleados con la finalidad de brindar un servicio de calidad y experiencia para personas que vivan cerca de la ZMG.

\section{RECOMENDACIONES Y LIMITACIONES}

Las limitaciones existentes es que no hay demasiada información acerca de las actividades que se pueden llevar a cabo en la Barranca del Río Santiago, de igual manera es importante mencionar que algunos lugares de esta zona no están en condiciones aptas, pues se encuentran contaminadas o deterioradas. Por ello, se recomienda que se pueda invertir una cierta cantidad de dinero para la restauración de algunas zonas con el fin de que poco a poco se vaya habilitando, para que pueda ser un atractivo turístico potencial para los habitantes de la zona metropolitana de Guadalajara, de igual manera generar más empleos. Asimismo, se pueden sumar más temas de investigación para abarcar temas de restructuración de desarrollos naturales y planes de negocios para el sector ecoturístico, entre otros.

\section{REFERENCIAS}

Anónimo, (s. f.), 10 problemas que enfrenta el turismo en diferentes ciudades de México. Entorno Turístico. Recuperado de http://www.entornoturistico.com/10-problemas-que-enfrenta-el-turismo-en-diferentes-ciudades-de-mexico/

Aldaz, P. (2016), La CDMX ya es una marca registrada. El universal. Recuperado de http://www.eluniversal. com.mx/articulo/metropoli/df/2016/01/27/lacdmx-ya-es-una-marca-registrada.

Aragón, M. (2014), Análisis del ecoturismo como alternativa de desarrollo sustentable en Latinoamérica (México, Costa Rica y Ecuador). El Colegio de la Frontera Sur. Université de Sherbrooke.

Armas, A. (2007), EN TORNO A LA MERCADOTECNIA URBANA: REORGANIZACIÓN Y REIMAGINACIÓN DE LA CIUDAD. REVISTA BIBLIOGRÁFICA DE GEOGRAFÍA Y CIENCIAS SOCIALES. Recuperado de http://www. ub.edu/geocrit/b3w-712.htm

Aventura en Zapopan. Viajes Huaxtla. Recuperado de http://www.huaxtla.org/index.php

Báez, L., Acuña, A. (1998), Guía para las mejores prácticas de ecoturismo en las Áreas Protegidas de Cen- 
tro América. Turismo \& Conservación Consultores, S.A. Recuperado de http://www.bio-nica.info/biblioteca/Baez\&Acuna\%201998.pdf

Benko, G. (2000), Estrategias de comunicación y marketing urbano. SciELO. Recuperado de https:// scielo.conicyt.cl/scielo.php?script=sci_arttext\&pid=S0250-71612000007900004.

Betancourt, Y. E. (2008), EL CITY MARKETING COMO UNA OPCION ESTRATEGICA DE MERCADOS. UNIVERSIDAD NACIONAL DE COLOMBIA. Recuperado de http://www.bdigital.unal.edu.co/1949/1/yesideduardobetancourt.2008.pdf

Bielsa, M. (2016), City Branding, el marketing de la cuidad. Mique. Recuperado de http://www.mique.es/citybranding-el-marketing-de-la-ciudad/

Catellanos, C. (2015), EVALUACIÓN DE LOS RECURSOS TURÍSTICOS CON VOCACIÓN ECOTURISTICA Y CARACTERIZACIÓN DE LA DEMANDA TURÍSTICA EN LAS ZONAS DE USO PÚBLICO DE LA RESERVA FORESTAL PROTECTORA DEL CERRO QUININÍ (TIBACUYCUNDINAMARCA). Colciencias. Recuperado de http://repository.udistrital.edu.co/bitstream/11349/2383/1/ CastellanosMenjuraClaudiaPatricia2015.pdf

Consultoria Mitosky, (2016), LOS ORGULLOS DE LOS MEXICANOS. Mexico opina. Recuperado de http:// consulta.mx/index.php/estudios-e-investigaciones/mexico-opina/item/821-los-orgullos-de-losmexicanos.

Comunicación social, eventos, gobierno noticias (2016), Presentan “Guadalajara Guadalajara”, la nueva marca cuidad. Gobierno de zapopan. Recuperado de https://www.zapopan.gob.mx/presentan-guadalajara-guadalajara-la-nueva-marca-ciudad/

CPTM. (2014), Programa Institucional 2014-2017. Consejo de Promoción Turística de México. Obtenido de http://www.cptm.com.mx/work/models/CPTM/ Resource/8183/8/images/Anexo_13_Programa_ Institucional_2014_2018_CPTM.pdf
CPTM. (2017), Internet. Consejo de Promoción Turística de México. Obtenido de http://www.cptm.com. $\mathrm{mx} /$ services/internet

García, M. (2016), La ciudad mexicana de Guadalajara presenta su nueva marca, basada en la famosa canción. Brandemia. Recuperado de http://www. brandemia.org/la-ciudad-mexicana-de-guadalajara-presenta-su-nueva-marca-basada-en-la-famosa-cancion.

Guadalajara Guadalajara. Recuperado de https://guadalajaraguadalajara.mx/

Guerrero, R. (2010), Ecoturismo Mexicano: la promesa, la realidad y el futuro. Un análisis situacional mediante estudios de caso. Redalyc. Recuperado de http:// www.redalyc.org/articulo.oa?id=193414423002.

Heredero, O., Chaves, M. (2015), LAS ASOCIACIONES "MARCA PRODUCTO" $Y$ "MARCA CIUDAD" COMO ESTRATEGIA DE "CITY BRANDING" Una aproximación a los casos de Nueva York, París y Londres. UNIVERSIDAD COMPLUTENSE DE MADRID. Recuperado de http://revistas.ucm.es/index.php/ ARAB/article/view/47857.

Hernández, E. (2014), Tesis. EL CITY MARKETING COMO ESTRATEGIA DE MERCADOS PARA EL DESARROLLO TURISTICO, CASO DE ESTUDIO IXTAPA DE LA SAL. UNIVERSIDAD AUTONOMA DE MEXICO. Recuperado de http://ri.uaemex.mx/bitstream/ handle/20.500.11799/30993/UAEM-FAPUR-TESIS-HERNANDEZ,\%20ESTEBAN.pdf?sequence=

Lascuráin, H. (1998), ELABORACIÓN DE INVENTARIOS DE ATRACTIVOS ECOTURÍSTICOS. Diana. Recuperado de http://www.ucipfg.com/Repositorio/MGTS/ MGTS14/MGTSV-07/tema2/Ceballos-Lascurain.pdf

Lloyd, C., Quercus, M., Medina, L. (s. f.). Cañon 7 cascadas. Jalisco Vertical. Recuperado de http://jaliscovertical.weebly.com/7-cascadas.html

Mancilla, E., Morales, A., Lugo, D. (2012), El papel imaginario urbano para la construcción de la marca 
de una cuidad. Imaginario Visual. Recuperado de http://eprints.uanl.mx/2551/1/El_papel_del_ imaginario.pdf

Marín, N. C. (2010), PROMOCIÓN TURÍSTICA A TRAVÉS DE LOS MEDIOS AUDIOVISUALES, CASO MEDELLÍN, COLOMBIA. Universidad Internacional de Andalucía. Recuperado de ttp://dspace.unia.es/ bitstream/handle/10334/757/0139_Gomez.pdf?sequence $=3$

Martínez, A. (2006), Creación de una marca de cuidad. Instituto Nacional de Administración Pública Centro de Estudios Locales. Recuperado de http:// bases.cortesaragon.es/bases/ndocumen.nsf/9ed3a8f9c37405f6c12570a6002b471d/24c0c410f75ea060c12575d8002abad1/\$FILE/creacin\%20marca\%20de\%20una\%20ciudad.pdf

Melo, J. (2013), El ecoturismo como desarrollo sustentable. UNAM. Recuperado de http://132.248.9.195/ ptd2014/enero/0707347/0707347.pdf

Navarro, D. (2015), Recursos turísticos y atractivos turístiCOS: CONCEPTUALIZACIÓN, CLASIFICACIÓN Y VALORACIÓN. Cuadernos del turismo. Recuperado de http://www.redalyc.org/pdf/398/39838701014.pdf

OMT, (2008), Entender el turismo: Glosario Básico. UNWTO. Recuperado de http://media.unwto.org/es/ content/entender-el-turismo-glosario-basico\#Turismo\%20interno

Portugal, M. (2008), Introducción al turismo. Transversal. Recuperado de http://enah.edu.ni/files/uploads/ biblioteca/902.pdf

Rebollo, N. (2012), Ecoturismo. Red Tercer Milenio. Recuperado de http://www.aliat.org.mx/BibliotecasDigitales/economico_administrativo/Ecoturismo.pdf

Rogel, I., Rojas, A., Ortega. S, (2011), EL TURISMO ALTERNATIVO COMO ESTRATEGIA DE CONSERVACIÓN DE
LA RESERVA DE LA BIOSFERA DE LA MARIPOSA MONARCA (2008-2010). Quivera. Recuperado de http:// www.redalyc.org/pdf/401/40119956006.pdf

Rojano, F. J. y Castilla, E. B. (2007), Presente y futuro de la comunicación estratégica en la ciudad. Fisec-estrategias Revista Académica del Foro Iberoamericano sobre Estrategias de Comunicación, No. 30. Recuperado de http://www.cienciared.com.ar/ra/ usr/9/318/fisec8m4_pp27_49.pdf

Romero, E. (2009). City-marketing, Marca-cuidad. Comunicaciones Estratégicas. Recuperado de http:// romeroads.blogspot.mx/2009/04/city-marketing-marca-ciudad.html?view=classic

Sancho, A. (2008), Introducción al turismo. E- unwto.org. Recuperado de http://moodle2.unid.edu.mx/dts_ cursos_mdl/lic/AET/PT/AM/01/Introduccion_turismo.pdf

SECTUR, (2014), AGENDAS DE COMPETITIVIDAD DE LOS DESTINOS TURÍSTICOS DE MÉXICO. Secturjal. Recuperado de https://secturjal.jalisco.gob.mx/sites/ secturjal.jalisco.gob.mx/files/u16/agenda_guadalajara.pdf

Valenzuela, R. (2016), Guadalajara, Guadalajara. Una marca sonora. Campaings \& Elections en español. Recuperado de https://www.campaignsandelectionsla.com/single-post/2016/10/01/Guadalajara-Guadalajara-Una-marca-sonora

Wallingre, N. (2011), EL DESARROLLO DE UN NUEVO DESTINO TURÍSTICO EL CASO DE LA CIUDAD DE VILLA ELISA, ARGENTINA. Dialnet. Recuperado de file:///C:/Users/DELL/Downloads/ Dialnet-EIDesarrolloDeUnNuevoDestinoTuristico-3915120.pdf

Wallingre, N., Toyos, M. (2010), Diccionario de turismo, hotelería y transportes, $2^{\mathrm{a}}$ ed., Buenos Aires, Universidad del Salvador 